\title{
Las trayectorias de las mujeres sin hijos en Uruguay
}

\author{
Ignacio Pardo* \\ Wanda Cabella** \\ Mathias Nathan ${ }^{\star * \star}$
}

El objetivo de este trabajo es analizar la población de mujeres uruguayas que finalizan su vida reproductiva sin hijos. Además de cuantificar el fenómeno, se busca responder las siguientes preguntas: ¿qué rasgos socioeconómicos las caracterizan?; ¿cuáles son las trayectorias típicas que conducen a la nuliparidez?; ¿en qué medida es el resultado de aspiraciones reproductivas frustradas o de la voluntad de no tener hijos? Para responder estas preguntas, se utiliza una estrategia de métodos combinados que integra datos cuantitativos y cualitativos. Los resultados muestran que la proporción de nulíparas descendió aproximadamente de $25 \%$ a $10 \%$, entre las nacidas entre 1900 y 1960, y que en los datos más recientes se detecta un incipiente incremento. Además, hay una mayor proporción de nulíparas entre las mujeres de nivel educativo alto. Finalmente, la tipologización de trayectorias hacia la nuliparidez definitiva reafirma la heterogeneidad de caminos y atributos: aunque se identificaron trayectorias guiadas por la intención de no tener hijos, un conjunto importante de mujeres llega a la nuliparidez como una consecuencia no buscada de la postergación de la maternidad.

Palabras clave: Fecundidad. Nuliparidez definitiva. Uruguay.

\footnotetext{
*Programa de Población, Universidad de la República, Montevideo, Uruguay (ignacio.pardo@cienciassociales.edu.uy; https://orcid.org/0000-0002-6185-1082).

** Programa de Población, Universidad de la República, Montevideo, Uruguay (wanda.cabella@cienciassociales.edu.uy; https://orcid.org/0000-0002-8522-7866).

${ }^{\star \star \star}$ Programa de Población, Universidad de la República, Montevideo, Uruguay (mathias.nathan@cienciassociales.edu.uy; https://orcid.org/0000-0002-0886-4183).
} 


\section{Introducción}

El aumento de la proporción de mujeres sin hijos es una de las facetas más importantes de los regímenes de baja fecundidad en los países desarrollados. Este fenómeno ha capturado la atención de la Demografía y de otras ciencias sociales, y ha estimulado la producción de una vasta literatura sobre sus causas y características. Por lo pronto, el creciente uso de métodos efectivos para el control de la fecundidad (casi universal en los países desarrollados) y el avance de la llamada revolución de género (ESPING-ANDERSEN; BILLARI, 2015) son los procesos que suelen invocarse para explicar el incremento del peso relativo de la nuliparidez entre las generaciones recientes.

Al mismo tiempo, hay discusiones acerca de cómo valorar el fenómeno que exceden las fronteras de la Demografía y generan debate público, enfrentando a quienes lo consideran negativo, por asociarlo al excesivo individualismo, a la precariedad de las relaciones conyugales o al posfamiliarismo de las sociedades actuales, con quienes ven en el aumento de la nuliparidez una potenciación de la autonomía de las mujeres (PETERSON, 2015). La distinción childless/childfree (BASTEN, 2009; GILLESPIE, 2003; MILLS et al., 2008) refleja tanto esta discusión valorativa como la necesidad de diferenciar la nuliparidez por elección de aquellas trayectorias de postergación de la maternidad que culminan involuntariamente en la nuliparidez (BERRINGTON, 2017), lo que a su vez nutre el debate sobre los límites biológicos de la fertilidad y el uso de las tecnologías de reproducción asistida (TE VELDE et al., 2012).

En cuanto a sus consecuencias demográficas, quienes se preocupan por la profundización del envejecimiento poblacional provocada por la baja de la fecundidad, ven con especial inquietud el aumento de las mujeres sin hijos, pero hay además una creciente discusión sobre las consecuencias en lo micro. Entre otras, el cambio en la identidad femenina en las mujeres sin hijos (GILLESPIE, 2003), el vínculo entre nuliparidez y transferencias intergeneracionales al final de la vida, ya sea en términos agregados o de bienestar personal, por la privación del apoyo que brindan los hijos durante la vejez (ALBERTINI; KOHLI, 2017; ALBERTINI; MENCARINI, 2014), o la salud física y mental de las mujeres nulíparas en comparación con las mujeres con hijos (GRAHAM, 2015; HUIJTS; KRAAYKAMP; SUBRAMANIAN, 2013).

Desde hace más de una década, Uruguay pertenece al grupo de países de baja fecundidad. La reducción registrada entre mediados de la década del noventa y mitad de la primera década de los 2000 llevó la tasa global de fecundidad (TGF) a un valor inferior a la a los dos hijos por mujer. Los datos de 2018 muestran que el indicador ha caído a 1,6 hijos por mujer (CABELLA; NATHAN; PARDO, 2019). El principal mecanismo demográfico que estimuló esta caída fue la reducción del número de nacimientos entre las mujeres que solían formar familias de tres o más hijos. Ni los cambios en la edad al primer nacimiento o en la proporción de mujeres que no tuvieron hijos al final de su ciclo reproductivo intervinieron significativamente durante esta fase de descenso de la fecundidad (NATHAN; 
PARDO; CABELLA, 2016). ¿Por qué es relevante entonces abordar el fenómeno de la nuliparidez en Uruguay?

Existen al menos dos razones para ello. En primer lugar, hay evidencia de un incipiente aumento de las mujeres sin hijos entre las cohortes que culminaron su vida reproductiva en años recientes, fenómeno que se verifica también en otros países latinoamericanos (LIMA; ZEMAN; SOBOTKA, 2018). En segundo lugar, se sabe que una parte de la población femenina, mayoritariamente de los estratos altos, ha comenzado a aplazar sistemáticamente la edad al primer nacimiento (NATHAN, 2015; NATHAN; PARDO; CABELLA, 2016), lo que se asocia al aumento contemporáneo de la nuliparidez definitiva (BERRINGTON, 2017; SOBOTKA, 2017; TE VELDE et al., 2012; WAGNER; HUININK; LIEFBROER, 2019).

Aunque la postergación de los nacimientos ha sido estudiada en Uruguay, no hay trabajos que aborden su potencial vínculo con la nuliparidez o que profundicen sobre las circunstancias y el proceso de toma decisiones reproductivas que pueden derivar en no tener hijos. También hay un vacío de evidencia respecto a la magnitud con que la población uruguaya ha modificado sus parámetros normativos en torno a la nuliparidez por elección. Nuestro objetivo es describir los atributos y las trayectorias de las mujeres uruguayas que llegan a los años finales de su vida reproductiva sin haber tenido hijos. Además, nos interesa especialmente saber en qué medida la nuliparidez es consecuencia de intenciones reproductivas frustradas o de una intención sostenida en el tiempo de no tener hijos.

\section{Antecedentes}

\section{La nuliparidez en los siglos XX y XXI}

En varios países desarrollados, la proporción de mujeres que no tienen hijos de forma definitiva ha crecido hasta llegar a niveles muy importantes; en casos como Suiza o España, representan entre una quinta y una cuarta parte de las cohortes que finalizaron su ciclo reproductivo en los primeros años de este siglo (KREYENFELD; KONIETZKA, 2017). Es una cifra alta, aunque no inédita: la evolución de la proporción de nulíparas en los países europeos no fue lineal en el transcurso del último siglo y las primeras décadas del siglo XXI; por el contrario, ha seguido una trayectoria marcada por fases de aumentos y descensos sostenidos. Lo inédito es que una parte de las nulíparas corresponda a mujeres que no tienen hijos por elección, en el contexto de competencia de la maternidad con otros proyectos vitales, especialmente laborales.

Sobotka (2017) resume el proceso de evolución de la nuliparidez europea en tres etapas históricas: un pico alto al comienzo del siglo XX, que corresponde a las cohortes de nacimiento entre 1880 y 1910 ; una caída posterior protagonizada por las cohortes de 1910 a 1945, y un crecimiento sostenido en las cohortes nacidas después de la Segunda Guerra Mundial, aunque con gran heterogeneidad en sus niveles: por debajo del $10 \%$ en países como Polonia, Bulgaria y Rusia, y más del 20\% en Alemania, España, Holanda 
e Italia (MIETTINEN et al., 2015). Estas etapas describen la experiencia europea, pero el patrón se expande más allá de ese continente. Rowland (2007), por ejemplo, muestra que en Estados Unidos, Australia y Japón se registró el mismo patrón temporal en la evolución de la nuliparidez.

¿Qué se puede decir sobre la nuliparidez en Uruguay? Tres estudios recientes para varios países de América Latina, incluido Uruguay, indican que hay un aumento de las mujeres sin hijos en las cohortes nacidas a fines de la década del setenta (FANTA GARRIDO; SACCO, 2018; LIMA; ZEMAN; SOBOTKA, 2018; REHER; REQUENA, 2014). Al mismo tiempo, está ocurriendo un paulatino proceso de aplazamiento de la edad al primer nacimiento, liderado por las mujeres con mayor nivel educativo, que podría verse como un posible precursor del aumento de la nuliparidez. Además, las transformaciones recientes en la formación y disolución de uniones, en línea con los cambios distintivos de la segunda transición demográfica, configuran un contexto cambiante entre las relaciones conyugales y la fecundidad, generando trayectorias reproductivas más tardías y segmentadas en distintos episodios conyugales (CABELLA, 2006; FERNÁNDEZ SOTO, 2010, 2019).

Respecto a la tendencia histórica, un estudio retrospectivo realizado a partir del censo de 1975 (PELLEGRINO; POLLERO, 1998) evidenció la existencia de un período de expansión de la nuliparidez en Uruguay seguido de otro de retracción, de forma no muy diferente a la observada en los países europeos. Entre las cohortes nacidas a fines del siglo XIX la proporción de mujeres sin hijos llegó al 25\%, mientras que con el avance del siglo XX se registró una importante merma de la nuliparidez, liderada por las mujeres que nacieron en la década del veinte y, en paralelo, un aumento de las mujeres que tuvieron un solo hijo. Entre las cohortes que culminaron su vida reproductiva a mediados del siglo XX, la proporción de mujeres sin hijos rondaba el 10\%. La evolución del fenómeno en Uruguay puede observarse con datos censales, con los que se observa su reducción, estabilización, e incipiente aumento en la sucesión de las cohortes de mujeres de al menos 45 años (Gráfico 1). Dado que se presenta información de supervivientes a la fecha de los censos, la proporción de mujeres sin hijos puede estar afectada por los diferenciales de mortalidad al interior de cada cohorte. En este sentido, las mujeres nacidas en la primera mitad del siglo estuvieron expuestas a una mayor probabilidad de morir antes de superar la etapa reproductiva que las nacidas en los años posteriores, con lo cual se pueden generar algunas alteraciones en las cifras por efecto de un sesgo de selección. No obstante, al superponer la información de cuatro censos se obtuvieron cifras consistentes del porcentaje de nulíparas por cohorte, por lo que se puede considerar que los datos utilizados no presentan sesgos de consideración.

El contexto regional puede describirse parcialmente, porque los estudios sobre la nuliparidez en América Latina son escasos. Se sabe que Brasil contaba en 2010 con 13\% de nulíparas entre 40 y 49 años, uno de los valores más altos en la región junto a Uruguay y que la desigualdad en la intensidad del fenómeno entre estratos sociales era especialmente pronunciada (CAVENAGHI; DINIZ ALVES, 2013). También sabemos que la proporción de mujeres sin hijos disminuyó en casi todos los países de América Latina a partir de las rondas 
censales de 1970, a excepción de aquellos, como Ecuador, que partieron de niveles muy bajos y, a diferencia de la tendencia promedial de la región, registraron un aumento de la proporción de mujeres nulíparas definitivas en las sucesivas rondas censales (BINSTOCK; CABELLA, 2019).

GRÁFICO 1

Proporción de mujeres de 45 años o más sin hijos, según cohorte de nacimiento Uruguay - 1975-2011

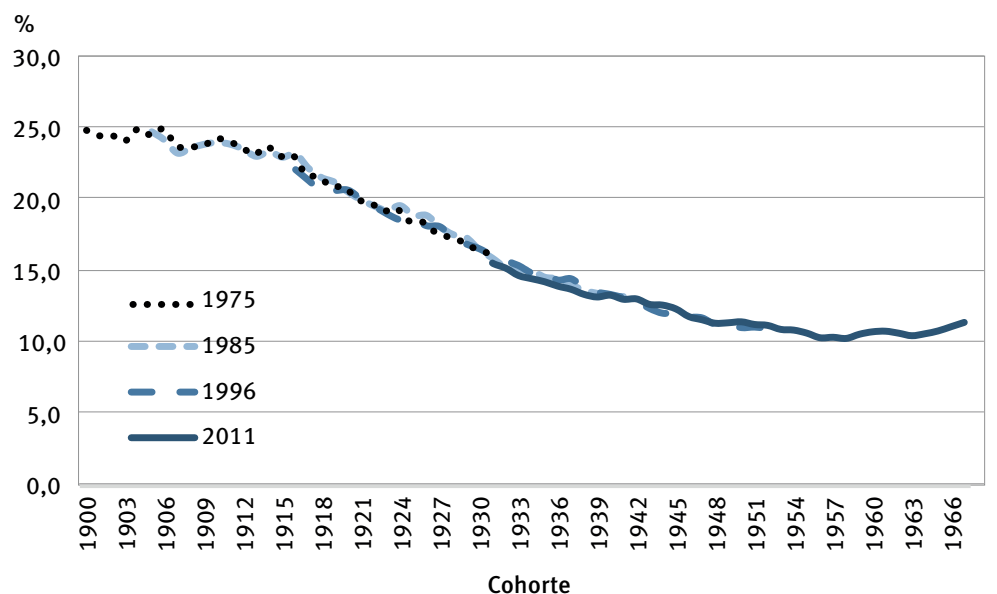

Fuente: Censos de población de 1975, 1985, 1996 y 2011. Se suavizaron las curvas en uno de los censos aplicando medias móviles trianuales.

En suma, la proporción de mujeres nulíparas osciló en diversas regiones del mundo, con distintos niveles de desarrollo. Aunque con diferentes ritmos e intensidades, el patrón fue similar: la nuliparidez aumentó, descendió y eventualmente volvió a aumentar. Mientras los dos primeros movimientos acompañaron los cambios de la fecundidad propios de la primera transición demográfica, ${ }^{1}$ el aumento reciente de las mujeres nulíparas, en especial en los países que han alcanzado valores muy altos, parece responder a nuevos esquemas valorativos de la maternidad y a dificultades de conciliación entre trabajo y familia. En este contexto, el valor de los ideales reproductivos, su traducción en intenciones y finalmente su realización o incumplimiento son componentes medulares de los procesos de toma de decisiones reproductivas asociados a la nuliparidez.

\footnotetext{
${ }^{1}$ En el caso de Uruguay, Pellegrino y Pollero (1998) caracterizaron el descenso de la nuliparidez como democratización en el acceso a la maternidad, en el marco de un aumento de las mujeres viviendo en pareja.
} 


\section{Las intenciones reproductivas y la nuliparidez voluntaria e involuntaria}

Las explicaciones en torno al aumento reciente de la nuliparidez son variadas. Tanturri et al. (2015) las ordenan en: perspectivas culturales, como la teoría de las preferencias o la segunda transición demográfica; perspectivas económicas en torno a teorías como la de elección racional o incertidumbre; perspectivas de género; y perspectivas de la demografía evolutiva, dentro de la que ubican hipótesis como las restricciones del mercado matrimonial, el decaimiento de las redes de apoyo a la crianza o la relación problemática entre búsqueda de ascenso social y cantidad de hijos. En cualquier caso, al explorar los factores explicativos de la nuliparidez se debieran distinguir las dos dimensiones del fenómeno: la nuliparidez voluntaria e involuntaria.

Inicialmente, esta distinción fue una forma de diferenciar la nuliparidez que respondía a causas biológicas de la que no. En su versión actual, es una medida de concreción de intenciones o ideales reproductivos, en cuya medición se ha avanzado recientemente (BEAUJOUAN; SOLAZ, 2016; KELLY, 2009; KREYENFELD; KONIETZKA, 2017; SOBOTKA; BEAUJOUAN, 2014). El carácter voluntario de la nuliparidez depende de si se quería o no tener hijos, pero para determinarlo con preguntas de encuesta se debe decidir si querer hijos refiere a ideales o a intenciones de fecundidad. El ideal de nuliparidez (esto es, una cantidad ideal de hijos igual a cero) es relativamente raro (SOBOTKA; BEAUJOUAN, 2014), mientras que no tener intenciones de tener hijos no lo es tanto. La diferencia entre ideales e intenciones puede deberse a que la pregunta sobre los ideales de fecundidad conduce a responder imaginando un escenario sin restricciones, mientras que las intenciones se manifiestan como la propensión a tener un hijo en el escenario más probable (CASTERLINE; EL-ZEINI, 2007). Este último es el indicador más utilizado, más allá de su volatilidad a lo largo del curso de vida (KUHNT; TRAPPE, 2016).

La volatilidad no se debe solamente a que las intenciones de tener un hijo pueden disminuir con el envejecimiento individual, sino a que se transforman a medida que se modifica la situación conyugal o la laboral. Para agregar complejidad, las personas ajustan sus preferencias a una suerte de cálculo de la probabilidad de que se concreten, por lo que del mismo modo que tener un hijo adicional puede empujar al alza las intenciones, acercarse al final de la vida reproductiva sin tenerlos puede también ajustarlas a la baja, lo que se ha reportado incluso antes de los treinta años (HAYFORD, 2009; IACOVOU; TAVARES, 2011; KUHNT; TRAPPE, 2016). Hasta esa etapa de la vida, las razones para la postergación pueden ser variadas: las más frecuentes son la educación y el empleo femenino, pero aparecen también los cambios de valores, la inestabilidad conyugal o las condiciones de la economía, las políticas sociales o la vivienda (MILLS et al., 2011). En todo caso, se ha observado que la mayoría de las mujeres nulíparas desearon un hijo en algún momento de su trayectoria y con el paso del tiempo ese deseo se fue desvaneciendo (CRAIG et al., 2014; IACOVOU; TAVARES, 2011). Esto podría reflejar, entre otras cosas, la importancia de las preferencias adaptativas en la medición de intenciones a edades avanzadas del ciclo reproductivo. 
Buhr y Huinink (2017) han propuesto cinco mecanismos como motor del ajuste en este tipo de trayectorias en las que la nuliparidez se vuelve voluntaria al final de la vida reproductiva: adaptación al estilo de vida sin hijos; resignación ante los escollos encontrados en el camino a la maternidad; disminución de la fertilidad; baja influencia de los pares en las presiones para tener hijos; y bajo nivel de persistencia en la búsqueda de metas personales como la maternidad. Esta fluidez entre nuliparidez voluntaria e involuntaria se reitera en la literatura sobre intenciones reproductivas, en la que la certeza absoluta y la ambivalencia prolongada son parte de un continuo que refleja que hay cambios frecuentes, o quizás como apuntan Ní Bhrolcháin, Beaujouan y Berrington (2010) - responda a que no se trata de que los eventos del curso de vida modifiquen intenciones, sino que estas nunca fueron más que una idea difusa, y ciertos eventos ayudaron a (no) concretarlas. En un estudio realizado a mujeres sin hijos en los Estados Unidos, se encontró que el $56 \%$ ya tenía la expectativa de no tener hijos a los treinta años, mientras que el resto llegó a la nuliparidez tras trayectorias diversas que en principio no excluían la maternidad (GEMMILL, 2019). En cualquier caso, esto no anula la utilidad de la distinción, elemental para el estudio de este tema.

Entre otras cosas, esta distinción genera una valoración distinta en términos de derechos reproductivos. Si no se tuvieron hijos porque no se los quería, se está cumpliendo con tener el número de hijos decidido: cero. Otra es la discusión ante la nuliparidez involuntaria. Finalmente, la distinción importa porque remite a agendas de investigación con énfasis distintos. En las nulíparas voluntarias, tiene el máximo interés conocer cómo se procesa el rechazo a la pérdida de identidad y las obligaciones y restricciones de la vida con hijos, así como la atracción por el estilo de vida sin hijos y su promesa de mayor autonomía personal y conyugal (BASTEN, 2009; GILLESPIE, 2003). Los temas asociados a la nuliparidez involuntaria tienen mayor relación con el vínculo entre las mujeres y el reloj biológico de la fertilidad (ESTEVE; TREVIÑO, 2019), más allá de su ampliación vinculada al aumento de las tecnologías de reproducción asistida.

\section{La dimensión socioeconómica de la nuliparidez}

La creciente acumulación en torno a los factores asociados a la nuliparidez definitiva permite conocer la estratificación del fenómeno, frecuentemente, relevada en términos de estratos educativos y organizada en dos hipótesis: a) los estratos de mayor educación tienen una mayor proporción de mujeres sin hijos; b) la relación entre estrato educativo y nuliparidez tiende a presentar forma de $U$, dado que hay también una mayor propensión a la nuliparidez de aquellas mujeres de educación muy baja.

La relación positiva entre nuliparidez definitiva y alto nivel educativo es una tendencia firme en muchos países occidentales (BEAUJOUAN; BRZOZOWSKA; ZEMAN, 2015; HAYFORD, 2013), más allá del debate en torno a los mecanismos: un mayor costo de oportunidad, una mayor independencia económica y autonomía personal (en parte por el efecto mediador de la menor propensión a casarse), el aplazamiento derivado de carreras educativas 
más largas o la orientación normativa a un ensanchamiento de las oportunidades de vida (NEYER; HOEM; ANDERSSON, 2017). Sin embargo, la experiencia de algunos países, como los nórdicos y los de Europa Oriental muestra que la relación no es siempre lineal. En cuatro países nórdicos, por ejemplo, el aumento reciente de la nuliparidez entre las mujeres de niveles educativos medio y bajo contribuyó a reducir la brecha educativa (JALOVAARA et al., 2018), y para Estados Unidos se ha observado la relación en U, estimando que algo más de una cuarta parte de las nulíparas lo son «por pobreza», mientras que para el resto opera un «alto costo de oportunidad de ser madres» (BAUDIN; CROIX; GOBBI, 2015). Otros estudios, de enfoque más original, vinculan la nuliparidez con el área de estudios más que con el nivel educativo (NEYER; HOEM; ANDERSSON, 2017; VAN BAVEL; JAN KOK, 2010).

La fuerza de la relación entre nivel educativo y nuliparidez es otra dimensión del tema. En estudios comparativos entre países desarrollados, se ha constatado que la relación es débil (BEAUJOUAN; BRZOZOWSKA; ZEMAN, 2016). Por cierto, no todos los países debieran comportarse como los desarrollados. En Brasil, uno de los pocos países latinoamericanos para los que tenemos estudios en profundidad, se observa una proporción mayor de nulíparas entre las mujeres de los estratos más altos, pero no la forma en U que implicaría valores más altos también entre las de nivel educativo más bajo (CAVENAGHI; DINIZ ALVES, 2013).

La estratificación del fenómeno tiene lugar a partir del vínculo de lo educativo con las trayectorias laborales y conyugales, y su nivel de incertidumbre (BERRINGTON; PATTARO, 2014; BUSETTA; MENDOLA; VIGNOLI, 2019; HAYFORD, 2013). Específicamente, las trayectorias conyugales fragmentarias o asociadas a la no existencia de pareja estable son determinantes importantes de la nuliparidez (JALOVAARA; FASANG, 2017; KREYENFELD; KONIETZKA, 2017), sobre todo cuando la edad a la primera unión es tardía. Esto refuerza la idea de la nuliparidez definitiva como el resultado de un proceso que se extiende en el tiempo, tras decisiones nutridas por la acumulación de contingencias y por la influencia mutua de las decisiones tomadas en distintas dimensiones de la vida. También invita a incorporar con mayor fuerza factores relacionados con la dinámica conyugal, como los desacuerdos en torno al proyecto reproductivo al interior de la pareja.

Finalmente, la estratificación de la nuliparidez no resulta de una interacción en el vacío entre atributos individuales y resultados de fecundidad, sino que tiene lugar en un contexto de nivel macro, cuyas características se ha intentado medir, con las dificultades del caso. Las variables más mencionadas como candidatas a mediar la relación entre atributos individuales y nuliparidez son los arreglos institucionales (NEYER; HOEM; ANDERSSON, 2017), la matriz de políticas familiares (TOCCHIONI, 2018) o las normas sociales, incluida la aceptación que existe en cada país hacia la nuliparidez voluntaria (MERZ; LIEFBROER, 2012). Como se ha visto, la acumulación sobre los factores asociados a la nuliparidez se vincula con lo que sabemos sobre la relación entre fecundidad y estratificación social en términos generales, aunque, tal como apuntan Tanturri et al. (2015), el tema necesita de una perspectiva singular, por lo que el desarrollo teórico y la exploración empírica desde métodos cuanti y cualitativos necesitan potenciarse. 


\section{Datos y métodos}

En este trabajo adoptamos una estrategia de métodos combinados, utilizando la evidencia recogida en dos componentes de investigación: uno cuantitativo, a partir de la explotación de los censos nacionales de población y la Encuesta Nacional de Comportamientos Reproductivos (Encor) de $2015 ;^{2}$ otro cualitativo, con fuente en entrevistas colectivas a mujeres nulíparas de 35 años en adelante.

La combinación de métodos nos permite integrar datos que describen atributos de la población con datos que aportan significados para interpretar tales atributos y narrativas vitales de las trayectorias vinculadas a la nuliparez. Esta estrategia permitirá abordar tres ejes de análisis:

- examinar la evolución de la proporción de mujeres sin hijos al finalizar la etapa reproductiva en Uruguay para las nacidas entre 1900 y 1968 (cuyos resultados fueron reseñados más arriba), así como sus características sociodemográficas básicas, a partir de los censos de población de Uruguay de 1975, 1985, 1996 y 2011;

- revisar las intenciones reproductivas de mujeres de 20 a 44 años sin hijos, a partir de la Encor de 2015;

- construir una tipología de trayectorias hacia la nuliparidez, con resultados de entrevistas colectivas, que permiten distinguir tipos de relación entre trayectorias conyugales, laborales y reproductivas, y aspiraciones reproductivas de las mujeres.

La combinación de métodos se hizo a dos niveles. Por un lado, por la complementariedad de la evidencia construida en uno y otro componente de investigación; por otro, por el uso de los resultados de encuesta para la conformación del diseño muestral del componente cualitativo. En la estrategia original se preveía la organización de tres entrevistas colectivas a mujeres nulíparas seleccionadas de la muestra de la Encor. Debido a las dificultades para recontactar a las encuestadas en 2015 y, en otros casos, para hacer efectiva la participación de encuestadas, la muestra quedó finalmente compuesta por diez mujeres previamente encuestadas en la Encor y por ocho mujeres reclutadas a posteriori a través de la técnica de bola de nieve. El trabajo de campo cualitativo se desarrolló durante el primer semestre de 2018. Las mujeres fueron convocadas al local de la Facultad de Ciencias Sociales (Universidad de la República, Uruguay) y las entrevistas fueron guiadas por dos

\footnotetext{
2 La Encuesta Nacional de Comportamientos Reproductivos fue realizada telefónicamente a una muestra compuesta por personas seleccionadas de una submuestra aleatoria de los hogares encuestados por la Encuesta Continua de Hogares $(E C H)$-la encuesta oficial de hogares de Uruguay- de 2015. El universo de la Encor estuvo conformado por las mujeres y varones de entre 15 y 44 años, residentes en hogares particulares en localidades urbanas de todo el territorio nacional. La muestra efectiva fue de 3487 personas (1583 varones y 1904 mujeres) entrevistadas entre noviembre de 2015 y marzo de 2016.
} 
expertas en la conducción de la técnica de entrevista colectiva. ${ }^{3}$ Todas las entrevistadas eran residentes de Montevideo y su área metropolitana. ${ }^{4}$

La pauta de entrevista fue acordada a lo largo de varias sesiones de discusión y lecturas del equipo de investigación en torno al fenómeno de la nuliparidez. Luego de haber sido validada por el grupo de investigación, fue discutida con las profesionales encargadas de guiar los grupos y se le hicieron ajustes en función de las experiencias recogidas en la primera entrevista colectiva. En suma, la entrevista grupal fue estructurada en función de las categorías de interés que surgieron del proceso teórico de investigación y ajustada a partir de las nuevas categorías y temas emergentes del trabajo empírico.

\section{Resultados}

Evolución de la nuliparidez según características sociodemográficas e intenciones reproductivas en Uruguay

Los datos confirman la existencia de una asociación positiva entre nuliparidez y nivel educativo alcanzado para el caso de Uruguay. Entre las mujeres de nivel bajo (educación primaria completa o menos) o medio (educación secundaria incompleta o completa) hay una menor proporción de nulíparas que entre las mujeres de nivel alto (estudios terciarios) (Gráfico 2). Así, la hipótesis que se sostiene entre las mujeres uruguayas es la de una relación lineal, en la que a mayor nivel educativo mayor la probabilidad de nuliparidez. Este patrón es estable entre las mujeres que terminaron su vida reproductiva de acuerdo con los datos de los censos de población desde 1975 hasta 2011.

Un aspecto que vale la pena destacar es que, entre las mujeres de nivel educativo alto, la proporción de nulíparas aumentó entre 1996 y 2011. Esto indica posiblemente que el estrato educativo más alto lidera la reversión del descenso de la nuliparidez, que se constata en los análisis censales a partir de las generaciones nacidas en la década del setenta, un fenómeno observado también en otros países de la región en la población total (FANTA GARRIDO; SACCO, 2018). Aunque el aumento es muy modesto, el comportamiento de las mujeres con mayor educación es claramente disonante respecto al de sus pares pertenecientes a otros estratos, en los que las reducciones son importantes y se sostienen hasta el último censo. Por otra parte, son también las mujeres con más años de educación quienes han postergado sistemáticamente la edad al primer hijo, al tiempo que entre las mujeres con nivel educativo bajo o medio no se registraron cambios en este indicador, lo que redundó en un ensanchamiento de la brecha educativa en la edad al primer hijo (NATHAN,

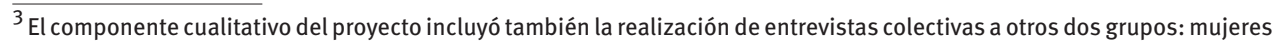
y varones indecisas/os respecto a la posibilidad de tener un segundo hijo; y mujeres que consideraban que habían tenido un número mayor que el deseado. En total el trabajo de campo cualitativo recogió las experiencias y opiniones de 59 personas residentes en Montevideo y su área metropolitana.

${ }^{4}$ Se cumplieron todas las normas habituales estandarizadas y las estipuladas por la Universidad de la República respecto a los estándares éticos del trabajo cualitativo y a la información relevada en entrevistas. Por la naturaleza del estudio, no se exigió la aprobación de un comité de ética.
} 
2015). Dado que la postergación está vinculada a una mayor probabilidad de nuliparidez involuntaria, es factible que las brechas continúen reforzándose en los próximos años.

\section{GRÁFICO 2}

Porcentaje de mujeres nulíparas en el grupo de edad de 45 a 49 años, por nivel educativo Uruguay - 1975-2011

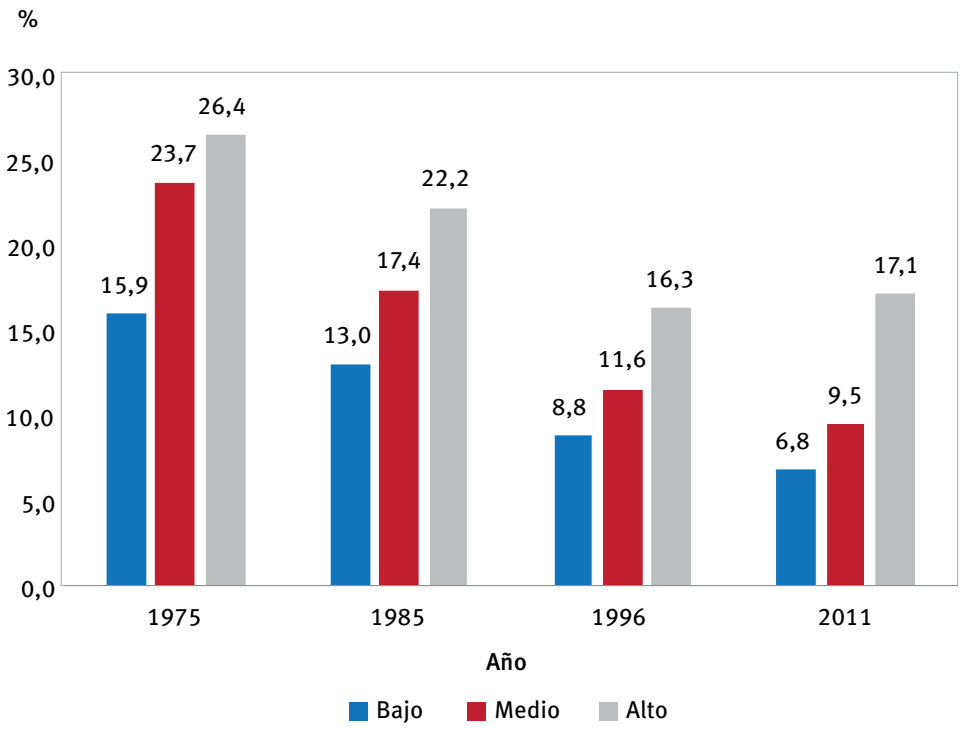

Fuente: Elaboración propia a partir de microdatos censales.

Otro punto de partida de la literatura sobre el tema es el vínculo entre las trayectorias de no haber vivido nunca en pareja, no encontrar la pareja adecuada con la cual tener hijos o haber pasado por una o más separaciones conyugales, con una mayor probabilidad de nuliparidez definitiva. A partir de los datos censales de Uruguay, se constata que, entre las mujeres nunca unidas, la proporción de nulíparas ha sido generalmente muy alta, aunque se observa su disminución con el correr del tiempo. A su vez, es escasa y decreciente la proporción de mujeres que están unidas o han estado alguna vez en unión (viudas, separadas y divorciadas) y culminan su vida reproductiva sin haber tenido hijos. En la globalidad del período considerado, menos de $10 \%$ de las mujeres censadas de entre 45 y 49 años, que pasaron por al menos un episodio conyugal, terminó su ciclo reproductivo sin haber tenido uno o más hijos (Gráfico 3).

Más allá de estas diferencias, la reducción de la proporción de nulíparas entre las mujeres nunca unidas amerita un análisis que excede el alcance de este trabajo: podría estar reflejando un debilitamiento de las barreras sociales existentes frente al ejercicio de la maternidad sin pareja o simplemente ser el resultado de cambios en la composición interna de este grupo de mujeres, entre otros posibles factores explicativos. De todos modos, para la mayoría de las personas, la unión conyugal sigue siendo el contexto más adecuado para concebir hijos (HAYFORD, 2013). Ante la parquedad de los datos censales, 
será el análisis cualitativo el que permita agregarle más detalle a los vínculos entre trayectoria conyugal y nuliparidez.

\section{GRÁFICO 3}

Porcentaje de mujeres nulíparas en el grupo de edad de 45 a 49 años, por estado conyugal (alguna vez en unión)

Uruguay - 1975-2011

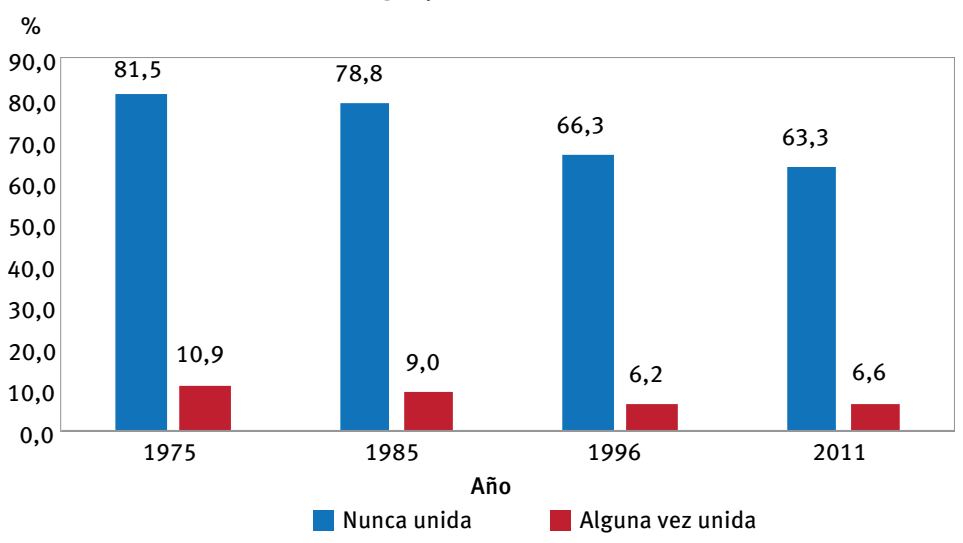

Fuente: Elaboración propia a partir de microdatos censales.

Finalmente, los datos permiten relacionar las intenciones reproductivas con la nuliparidez. Como se dijo antes, preguntar acerca del tamaño ideal de la descendencia («Si pudiera volver atrás, al momento en que no tenía hijos, y elegir la cantidad ideal...») suele disparar una respuesta asociada a un contexto sin restricciones más que uno de intenciones reales. Por ese motivo se suele contrastar el comportamiento reproductivo con las preguntas acerca de intenciones de fecundidad de cada hijo ("¿Piensa tener un hijo en el futuro?»). Utilizando ambas preguntas, los resultados muestran que la amplia mayoría de las mujeres tiene un ideal de uno o más hijos, con proporciones decididamente bajas de quienes declaran que no desean descendencia y dando como resultado un promedio algo superior a los dos hijos para casi todas las edades reproductivas. No obstante, al medir las intenciones y ya no los ideales se observó que la voluntad de tener un hijo entre las mujeres nulíparas va disminuyendo conforme aumenta su edad (Gráfico 4). Esto va en la misma línea de lo comprobado recientemente en otros contextos (BEAUJOUAN et al., 2019).

El análisis de la evolución conjunta de estas variables a lo largo de las edades sugiere que las intenciones reproductivas varían durante el curso de vida. Esta variación puede responder a situaciones de diversa índole, desde las restricciones dadas por el límite biológico de la fecundidad hasta la propia fluidez de las intenciones entre quienes comienzan a valorar las ventajas de no tener hijos. 
GRÁFICO 4

Proporción de mujeres de entre 15 y 44 años sin hijos que piensa tener hijos en el futuro y promedio ideal de hijos, por grupo de edad

Uruguay - 2015

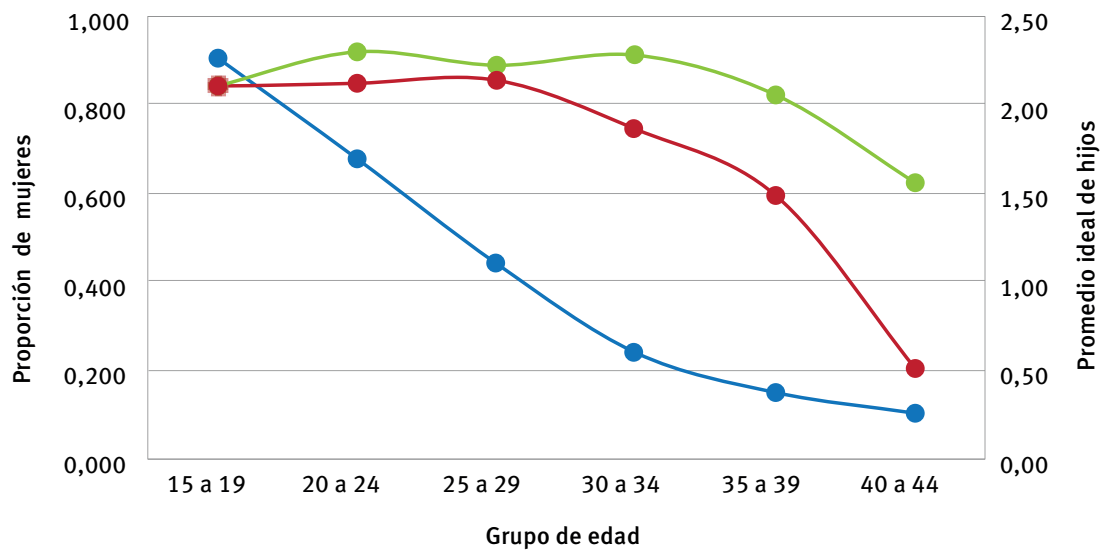

- Mujeres sin hijos $\quad \longrightarrow$ Piensa tener hijo(s) en el futuro $\quad$ Promedio ideal de hijos

Fuente: Elaboración propia a partir de datos de Encor de 2015.

En todo caso, las trayectorias que llevan a las mujeres hacia la nuliparidez no pueden describirse adecuadamente con datos de encuesta. Recurrir a evidencia cualitativa con la cual reconstruir retrospectivamente tales trayectorias permitirá interpretar con mayor sustento empírico los datos cuantitativos y las hipótesis que estos sugieren, así como cartografiar la heterogeneidad de la población de nulíparas. En palabras de Rybinska y Morgan (2019, p. 26, traducción nuestra) «ni siquiera la mejor base de datos longitudinal puede captar la idiosincrasia de las vidas vividas».

\section{Tipos de trayectorias hacia la nuliparidez}

El componente cualitativo de la investigación permitió identificar una tipología de trayectorias asociadas a la culminación de la vida reproductiva sin haber tenido hijos. Para eso, es tan necesario detectar preferencias o ideales estables en torno a la fecundidad como recrear las condiciones en las que las intenciones reproductivas más concretas fueron desplegándose a lo largo de la vida en respuesta a los contextos conyugales, laborales, económicos o de acumulación educativa. Los tipos de trayectorias identificados son los siguientes:

1. de preferencias estables por rechazo a roles tradicionales;

2. postergadoras por inestabilidad conyugal;

3. postergadoras extremas;

4. postergadoras por inestabilidad laboral o económica;

5. postergadoras por desarrollo personal (educativo y laboral). 
En el primer tipo hay una preferencia sostenida en el tiempo por no tener descendencia, mientras que en los últimos cuatro el factor que lleva a las mujeres a llegar a los últimos años de vida reproductiva sin hijos es la postergación de la maternidad. Así, ambos grupos de trayectorias pueden asociarse a la nuliparidez voluntaria e involuntaria, respectivamente. Si bien la tipología no tiene pretensiones de exhaustividad, describe las trayectorias principales que pudieron extraerse de la muestra de mujeres entrevistadas. El interés de la tipología radica además en que los tipos identificados tienen un correlato en varios de los temas identificados como clave en la acumulación bibliográfica sobre el tema y en algunos de nuestros resultados cuantitativos.

\section{- De preferencias estables por rechazo a roles tradicionales}

Este tipo de trayectoria se singulariza por una preferencia por el desarrollo personal y su contraposición con la maternidad. El desarrollo personal puede asociarse a variedad de metas, entre las que se destacan la carrera profesional, la realización de viajes, el compromiso con causas sociales y el tiempo de ocio individual o con familiares y amigos. La referencia a los roles tradicionales entre las mujeres asociadas a este tipo de trayectorias suele darse en términos del rechazo al proyecto de la maternidad, percibida como opción que socavaría la consecución de las otras metas personales. Más allá de que es posible que hayan forjado un discurso acorde a sus resultados reproductivos -recordemos que son narrativas retrospectivas, se percibe una postura elaborada frente a la maternidad: estas mujeres hicieron un balance de alternativas vitales que resultó en la presentación de sí mismas como mujeres que optaron por no ser madres de forma deliberada.

Además, aunque no se trate de un atributo que las defina plenamente, las trayectorias de estas mujeres están atravesadas por períodos de inestabilidad conyugal. Algunas han tenido dificultades a la hora de compatibilizar los intereses y proyecciones de su vida personal con los de la vida en pareja, en cierta medida por su negativa a convertirse en madres, pero también por su postura crítica frente a las conformaciones familiares o conyugales más clásicas.

- Aparte vos te sentás y te analizás y decís «Yo estoy bien así, a ver, ¿por qué me generaría algo... que no lo tengo por mí misma?» Y te miran como «Pobrecita, no consigue novio».

- Y con lo que no tenés hijos... a mí ya todo eso lo tengo más que repasado... (risas). A mí lo que me llama sobre todo... es que también hay diferencia, digamos, interclases... porque los sectores que somos medios y más educados podemos entender que la única realización de la mujer no es la maternidad, intelectualmente lo podemos entender. Pero los sectores más populares...

- Peor es que te digan «¿Yquién te va a cuidar?» (risas).

- ... yo, por ejemplo, estoy en una etapa [en la] que no me interesa lo que digan. Me parece que estar bien yo misma me alcanza y me sobra.

Los discursos de las mujeres nulíparas que se fundamentan en la renuencia a asumir los roles familiares tradicionales se vinculan con una mayor aceptación social de la diversidad 
de trayectorias, incluso de aquellas que cuestionan el valor de la maternidad (GEMMILL, 2019; KELLY, 2009; PETERSON, 2015; TOCCHIONI, 2018). Su aparición pone de manifiesto que existe un grupo de mujeres que valoran otros proyectos personales por encima de la maternidad y que están dispuestas a expresarlo aun cuando su entorno las repruebe o cuestione la autenticidad de su decisión. Las reacciones del entorno, a su vez, reflejan que la sociedad está dispuesta a tolerar que el resultado de una trayectoria puede culminar en la nuliparidez, pero no tanto a empatizar con la idea de que las mujeres pueden decidir no tener hijos. Esta es una discusión instalada en los países en los que la proporción de mujeres sin hijos ha aumentado (BASTEN, 2009).

Además de esta trayectoria, que contempla la ausencia de hijos como una elección fundada en sus intereses o su identidad femenina, detectamos un segundo tipo de preferencias estables, vinculado a la sobrecarga de cuidados familiares. Debido a que el sustento empírico es menos evidente que en la primera, decidimos no definirla como una trayectoria separada, pero tiene interés describirla. En este caso, la preferencia estable por no tener hijos se deriva de una carga de cuidados familiares que suprime la maternidad por la vía de los hechos más que por la adhesión a una postura. La posibilidad de cuidar hijos es vista como un costo excesivo en términos de tiempo, tanto que considerar la posibilidad de aumentar la carga asociada a las nuevas obligaciones del cuidado infantil es vista como una tarea imposible.

- Y también tengo siempre el rol de estar acompañando a mi hermano con síndrome de Down y todo eso.

Esta trayectoria es infrecuente y en cierta medida parece ser un resabio de etapas históricas en las que las familias reservaban alguno de sus miembros fuera del matrimonio y la fecundidad, en general mujeres, para cuidar de los familiares ancianos (PELLEGRINO, 1997). Siguiendo la tipología de Reher (1998), Uruguay puede incluirse en la categoría de lazos familiares fuertes, es posible entonces que la percepción del peso de las responsabilidades familiares prime por sobre las preferencias personales, en especial cuando hay miembros que demandan cuidados especiales.

\section{- Postergadoras por inestabilidad conyugal}

Este tercer tipo de trayectorias está conformado por mujeres que pasaron la mayor parte de su vida reproductiva en duda acerca de la posibilidad de tener hijos. La incertidumbre estuvo relacionada con la situación conyugal en cada una de las etapas de su curso de vida: en contextos conyugales estables, en los que ambos miembros de la pareja estuvieron interesados en la búsqueda de un embarazo, estas mujeres se mostraron dispuestas a tener su primer hijo, pero no en otros contextos (por ejemplo, como «madres solteras»).

Por ese motivo, una trayectoria vital signada por disoluciones de los vínculos conyugales, generalmente de lazos de corta duración, trae consigo condiciones no adecuadas para la maternidad. 
- Nunca fue... Yo no es que no tenga instinto maternal, digo, porque yo con mi sobrina la adoro, la llevo a todos lados, siempre que me voy de vacaciones la agarro y me la llevo conmigo... pero claro, es así, es que la tengo y es eso... ya sé que la puedo devolver (risas). Y tampoco se dio. A ver, si yo hubiera tenido una pareja y con esa pareja me hubiera sentido cómoda y esa pareja hubiera querido tener... hubiera tenido.

- Sí, eso se ve en montones de parejas. Pero también está en uno... no asumir todo, la mujer no tiene por qué ser mujer, ser madre. No es un rol establecido por nadie, eso cada uno lo decide. O sea, yo ya la dije a mi pareja "Esto es de a dos, no es de a uno, sino también es egoísta». Sí, vos tenés el hijo, que la madre lo cuide. No lo veo así.

Este tipo de trayectoria ha sido extensamente analizada en la bibliografía en torno a la nuliparidez en los países que tienen altas proporciones de mujeres que culminan su vida reproductiva sin haber tenido hijos (JALOVAARA; FASANG, 2017; MILLS et al., 2011; SOBOTKA, 2017). La inestabilidad conyugal es una característica de estas sociedades desde hace varias décadas y ha sido acompañada por la aparición de trayectorias de parejas cada vez más complejas (GUZZO, 2014). Las mujeres posponen la llegada del primer nacimiento hasta encontrar la situación de pareja que colme sus expectativas para llevar adelante su proyecto reproductivo, pero a la vez las expectativas sobre las características del cónyuge adecuado son progresivamente más difíciles de alcanzar. En el corto y largo plazo todas estas condiciones implican más exigencias respecto a la vida conyugal, lo que implica mayores probabilidades de conflicto y ruptura y en definitiva restringe los tiempos en los que las mujeres encuentran las condiciones conyugales adecuadas para tener hijos.

\section{- Postergadoras extremas}

Uno de los tipos de trayectoria que resulta más arriesgada en términos de cumplir con el deseo de ser madre en algún momento de la vida, es aquel que está marcado por postergaciones sucesivas de la maternidad. Las postergadoras extremas no solo aplazan el momento de buscar un embarazo sino también la propia decisión, que flota en algún momento indeterminado del futuro, a pesar de que sus trayectorias conyugales sean a menudo estables.

El nivel de contingencia al que queda sometido un proyecto de ser madre que no ha sido descartado ni emprendido, se vincula asimismo con la falta de información respecto a la merma de la fertilidad que sufren las mujeres que se va acercando a los cuarenta años. A esas edades, la postergación indefinida aumenta las probabilidades de que, en caso de buscar un embarazo en el futuro, puedan enfrentarse a la imposibilidad de concebir o a sufrir fracasos gestacionales (GEMMILL, 2019; TE VELDE et al., 2012).

\section{- Postergadoras por inestabilidad laboral y/o económica}

Como se señala en la literatura sobre intenciones reproductivas, la incertidumbre respecto a la estabilidad laboral o económica explica gran parte de la postergación (MILLS et al., 2011; SOBOTKA, 2010), al menos en ciertos contextos. Un tipo de trayectoria que puede asociarse a comportamientos de esta índole es aquel que está determinado por la confluencia entre 
motivaciones personales de mejora profesional y normas respecto al nivel de bienestar y estabilidad que es necesario alcanzar para enfrentar las responsabilidades de la crianza.

La confluencia de ambos factores, en este tipo de trayectorias, genera un comportamiento de aplazamiento de la maternidad que coloca a la intención de tener el primer hijo en un futuro en el que el empleo sería bien remunerado y altamente estable. Si la evaluación que hace la entrevistada acerca de sus posibilidades es sostenidamente negativa, es muy probable que el resultado sea la llegada a la etapa final de la vida reproductiva sin hijos.

- Sí, capaz que un poco más de ganas tuve... El tema siempre esperando la estabilidad económica, yo qué sé. A mí los treinta a los cuarenta se me volaron, fue una cosa increíble.

- La realidad ha sido económica, sobre todo. Si hubiera tenido una estabilidad... Si supiera... La plata siempre va a estar justa, con esto que pensamos hacer ahora, hay pila de ideas, pero no es que vamos a nadar en plata, saber que se pagan las cosas fáciles, saber que contás con esa plata por mes.

A diferencia de la categoría siguiente, en este grupo de mujeres la situación económica de la pareja juega un rol importante en la postergación que culmina en nuliparidez. Por supuesto, en la postergación de la maternidad vinculada a la inestabilidad laboral o económica existe una preocupación por la propia inserción en el mercado laboral, pero se privilegia un argumento que incluye algún grado de altruismo, en el sentido de que la postergación está fundada en la búsqueda de un contexto de crianza que colme las expectativas de consumo que se creen adecuadas para garantizar el bienestar de los hijos.

\section{- Postergadoras por desarrollo personal (educativo o laboral)}

Finalmente, en este tipo de trayectoria, también ampliamente descrita en la literatura sobre el tema, las decisiones de estas mujeres a lo largo de su vida reproductiva no están tan asociadas con la idea de estabilidad laboral sino con la de desarrollo personal a través de la carrera profesional (BUHR; HUININK, 2017; CRAIG et al., 2014; GILLESPIE, 2003). Estas preferencias dificultan la conciliación entre la vida laboral y familiar (HAKIM, 2003), y promueven la postergación del primer hijo hasta el momento de desarrollo máximo de los objetivos de carrera, en un contexto en el que los proyectos vinculados con la inserción en el mercado laboral son progresivamente más ambiciosos.

- Yo en realidad siempre quise ser contadora o licenciada en Marketing. De contadora no me fue bien en Facultad: ingresé varias veces, conflicto tipo de paros largos, abandonaba. Empecé a trabajar a los 21 ... poco tiempo, eso, de cursar alguna materia. $Y$ no me terminaba de concluir nunca. Entonces, hice Marketing en la UTU5 y para hacer la licenciatura solo es privada y no la puedo costear. Así que, ta, ahora me metí en Comunicación porque el año pasado envié un mail contando un poco lo que yo quería para ver si había algo dentro de la Facultad. Y me dijeron que sí, que había un área de publicidad. Y bueno, me enganché este año.

\footnotetext{
${ }^{5}$ Consejo de Educación Técnico Profesional-Universidad de la Trabajo del Uruguay.
} 
- Porque mi tía que me jode [...], ella tuvo seis hijos, ta, fantástico. Pero ella, por ejemplo, tuvo seis hijos, pero postergó toda su carrera, que hizo Enfermería, y no pudo seguir laburando que era lo que más le gustaba. Entonces, por un lado, me pregunta eso, pero por el otro sabe que me deslomé para poder tener una carrera. Entonces como que siempre ven la deficiencia, lo que ellos no pudieron.

- En realidad, yo lo que siempre prioricé fue mi carrera. Me llegué a recibir, carrera larga... y claro, después es como que te lleva a otra realidad. Me quiero recibir, me recibo... ¿y ahora?

La evidencia recogida en el componente cuantitativo acerca de la probabilidad diferencial de nuliparidez en mujeres con distinto nivel educativo generó algunas pistas sobre posibles trayectorias reproductivas que pronosticaban la nuliparidez definitiva. La relación causal entre acumulación educativa y nuliparidez no es clara y puede funcionar en ambas direcciones. Además, en el caso de los datos cuantitativos, el nivel educativo podría tener importancia en sí mismo o funcionar como proxy de otros atributos estructurales. Por todo eso, es útil observar con datos cualitativos este tipo de trayectoria, de manera de desentrañar al menos uno de los mecanismos que relaciona nivel educativo con nuliparidez.

La información cualitativa nos permitió profundizar en las opciones y los recorridos vitales de las mujeres que, por distintas combinaciones de motivos, preferencias y experiencias vitales, condujeron, voluntaria o involuntariamente a terminar su ciclo reproductivo sin hijos. Si bien el número de entrevistas no fue el ideal para ilustrar con mayor detalle las trayectorias que identificamos, detectamos narrativas que nos facilitaron la determinación de núcleos orientadores de distintas trayectorias. No podemos afirmar que estos núcleos sean privativos o singulares de cada trayectoria, pero en las narrativas de las entrevistadas es claro que tanto las decisiones como la postergación de las decisiones no tienen una razón única. Lo que encontramos son énfasis, elecciones discursivas para relatar las experiencias, que nos permitieron identificar los núcleos rectores que las entrevistadas priorizan en su curso vital como los precursores de la nuliparidez. La ambigüedad en el deseo de tener hijos y en las intenciones, la modificación de las expectativas en función de acontecimientos y valoraciones cambiantes, la difusa necesidad de elegir entre maternidad y otros proyectos, entre otros, se combinan de formas que ameritan más estudios para entender cómo su interacción culmina en explicaciones complejas de la nuliparidez.

Un aspecto que sí podemos confirmar para el caso uruguayo, en sintonía con los resultados de investigación en la literatura internacional, es la importancia de la estabilidad conyugal a lo largo de la vida y la distinción entre decisión firme y postergación (asociado a nuliparidez voluntaria/involuntaria) que marcó cada tipo de trayectoria. En relación con estos ejes, se pueden ubicar los seis tipos de trayectorias antes descritos (Cuadro 1). 
CUADRO 1

Representación de los grupos de trayectorias detectados en mujeres sin hijos

\begin{tabular}{|ccc|}
\hline & Decisión firme & Postergación \\
\hline Inestabilidad conyugal & Tipo 1 & Tipo 3 \\
\hline Estabilidad conyugal & & Tipo 4 \\
& & Tipo 5 \\
& Tipo 6 \\
\hline
\end{tabular}

Fuente: Elaboración propia.

\section{Discusión}

La evolución de la nuliparidez es uno de los fenómenos más relevantes del comportamiento demográfico en las poblaciones de baja fecundidad (BALBO; BILLARI; MILLS, 2013; SOBOTKA, 2017; TANTURRI et al., 2015). El aumento de las nulíparas en los países pioneros del descenso de la fecundidad y la heterogeneidad de su composición son referencias centrales en la construcción de expectativas teóricas acerca de la evolución de la fecundidad. Estos ejes pueden sustentar hipótesis sobre la evolución del fenómeno para aquellas poblaciones, como la uruguaya, en las que la baja fecundidad es más reciente y la proporción de nulíparas presenta aumentos apenas incipientes.

En este trabajo, los datos han mostrado la relación lineal entre educación y nuliparidez; la centralidad de las trayectorias conyugales en la explicación de la maternidad, y la persistencia de ideales de fecundidad cercanos al ideal de dos hijos entre las nulíparas, junto con el ajuste a la baja de sus intenciones al llegar a la última etapa de su vida reproductiva. También evidenció que las trayectorias hacia la nuliparidez son diversas, con pluralidad de caminos asociados a la postergación, que deriva en nuliparidez involuntaria. ¿Qué implicaciones tiene esto a la hora de especular acerca de tendencias futuras? Para empezar, si las tendencias se comportan dentro de lo previsible, los niveles de fecundidad total se estabilizarán en valores bajos y es plausible que al mismo tiempo aumente la nuliparidez definitiva. La manera en que este cambio se procese dependerá de varios factores.

En primer lugar, es esperable una profundización de la postergación de la maternidad, que ya está en marcha. Varias trayectorias típicas, como las de las postergadoras extremas o las de las postergadoras por desarrollo personal, podrían extenderse a sectores más amplios de la población, lo que aumentaría el riesgo de nuliparidez involuntaria. El mecanismo podría describirse como una serie de aplazamientos sistemáticos en la toma de decisiones reproductivas a lo largo de toda la vida fértil, enmarcados en una ausencia de la necesidad de concreción de un deseo reproductivo.

En segundo lugar, es razonable pensar que este tipo de trayectorias, atadas a la probabilidad de permanecer nulípara, seguirá distribuyéndose diferencialmente entre las mujeres de distinta condición socioeconómica. Aunque el descenso de la fecundidad adolescente observado en los últimos cuatro años tiene un efecto ligeramente igualador del calendario del primer hijo, en las edades más avanzadas de la vida reproductiva las brechas seguirán 
siendo importantes, entre otras cosas porque el acceso a la educación superior sigue estando estratificado entre sectores sociales (CABELLA; NATHAN; PARDO, 2019). Es cierto que el vínculo entre postergación y nuliparidez puede morigerarse por el acceso de los estratos más altos a tecnologías de reproducción asistida, pero por el momento este efecto es muy modesto y no es esperable que altere el patrón de heterogeneidad por estratos.

En tercer lugar, las políticas públicas han comenzado a intervenir en una dimensión que podría modificar otras trayectorias identificadas: las que derivan en nuliparidez por incertidumbre laboral o sobrecarga de cuidados. En la situación dilemática entre consolidar una carrera laboral competitiva o dedicar tiempo a la crianza de los hijos, media la acción del Estado en la provisión de cuidados que permitan conciliar vida familiar y trabajo, así como el ambiente cultural en torno al cambio en los roles de género. Se ha postulado la emergencia de un nuevo equilibrio entre las relaciones de género y la posibilidad de conciliación trabajo-crianza a partir del concepto de «revolución incompleta de género» (ESPING-ANDERSEN; BILLARI, 2015) de forma de ilustrar esa transición entre dos equilibrios, que culminaría con la recuperación de niveles de fecundidad más cercanos a dos hijos. De la velocidad de esa transición depende la disminución de la insatisfacción con la fecundidad en las mujeres y la magnitud de su incremento más allá de niveles bajos en términos agregados.

Así, se suele esperar que la transición hacia el modelo de doble proveedor traiga consigo mayor equidad de género y de ese modo el mecanismo utilizado por muchas mujeres para reducir su doble carga de trabajo deje de ser el ajuste a la baja de la fecundidad. Ahora bien, las esperanzas puestas en la capacidad de las políticas de corresponsabilidad de corregir la insatisfacción entre cantidad de hijos deseados y tenidos han sido a menudo demasiado optimistas. De todos modos, si las políticas, en especial las dirigidas a fortalecer el cuidado público infantil, tuvieran éxito, podrían reducir la cantidad de personas nulíparas por postergación o por falta de condiciones para compatibilizar crianza y otras dimensiones de la vida.

En cuarto lugar, hay otro tipo de factores que inciden en el posible aumento de la nuliparidez. Uno de ellos es el mercado matrimonial y la dinámica general del cambio familiar y las uniones conyugales. Entre otras cosas, la modificación del contexto percibido como legítimo para tener y criar hijos. Si la maternidad sin pareja se consolidara como una opción desde el comienzo de la decisión de tener un hijo, podría descender la proporción de nulíparas por inestabilidad conyugal. El escenario es probable a mediano plazo, aunque por el momento está muy arraigada la apuesta a tener hijos con una pareja estable, en un contexto de creciente inestabilidad conyugal. De esa contradicción surge la mayor probabilidad de nuliparidez. Sin embargo, hay otro factor clave que vincula fecundidad e inestabilidad conyugal: el aumento de disoluciones a edades cada vez más tempranas promueve la formación de nuevas parejas cuando aún es posible replantearse el proyecto reproductivo, e invita a estudiar con mayor detalle el potencial reproductivo de las segundas o terceras uniones. 
Finalmente, es necesario seguir la evolución del cambio cultural que legitima la norma por la cual no tener hijos no merece sanción social. Aunque tal proceso está en marcha, la ideología familista es aún importante y, como vimos, quienes prefieren no tener hijos a lo largo de toda su vida son una proporción mínima de las mujeres. Es posible que la tensión entre ambas fuerzas genere procesos de valuestretch (RODMAN, 1963), por los cuales los valores se adapten a las nuevas condiciones sin abandonar del todo el resto de las valoraciones, en apariencia contrapuestas. Es de esperar que tal proceso colabore en alguna medida con el aumento en la proporción de nulíparas voluntarias. Como fuere, el eventual incremento de la nuliparidez otorga una nueva oportunidad para que el estudio del comportamiento reproductivo conecte las restricciones estructurales y las orientaciones valorativas de las personas, dos factores que se influyen mutuamente.

\section{Referencias}

ALBERTINI, M.; KOHLI, M. Childlessness and intergenerational transfers in later life. In: KREYENFELD, M.; KONIETZKA, D. (ed.). Childlessness in Europe: contexts, causes, and consequences. [S.I.]: Springer, Cham, 2017. p. 351-368.

ALBERTINI, M.; MENCARINI, L. Childlessness and support networks in later life: new pressures on familistic Welfare States? Journal of Family Issues, v. 35, n. 3, p. 331-357, 2014.

BALBO, N.; BILLARI, F. C.; MILLS, M. Fertility in advanced societies: a review of research. European Journal of Population, v. 29, n. 1, p. 1-38, 2013.

BASTEN, S. Voluntary childlessness and being Childfree: the future of human reproduction. [S.I.]: University of Oxford, Viena Institute of Demography, 2009. (Working Paper, n. 5). Disponível em: https://www.spi.ox.ac.uk/fileadmin/documents/PDF/Childlessness_-_Number_5.pdf. Acesso em: 13 ago. 2015.

BAUDIN, B. T.; CROIX, D. de; GOBBI, P. E. Fertility and childlessness in the United States. American Economic Review, v. 105, n. 6, p. 1852-1882, 2015.

BEAUJOUAN, E.; BRZOZOWSKA, Z.; ZEMAN, K. Childlessness trends in twentieth-century Europe: limited link to growing educational attainment. Vienna: Austrian Academy of Sciences (ÖAW), Vienna Institute of Demography, 2015. (Vienna Institute of Demography Working Paper, n. 6). Disponivel em: https://www.oeaw.ac.at/fileadmin/subsites/Institute/VID/PDF/Publications/ Working_Papers/WP2015_06.pdf. Acesso em: 31 maio. 2019.

BEAUJOUAN, E.; BRZOZOWSKA, Z.; ZEMAN, K. The limited effect of increasing educational attainment on childlessness trends in twentieth-century Europe, women born 1916-65. Population Studies, v. 70, n. 3, p. 275-291, 21 ago. 2016.

BEAUJOUAN, E.; SOLAZ, A. Are family sizes of parents and children still related? Revisiting the cross-generational relationship over the last century. [S.I]: Institut National d'Etudes Démographiques (INED), 2016. (Documents de Travail, 223).

BEAUJOUAN et al. Declining realisation of reproductive intentions with age. Human Reproduction, v. 34, n. 10, p. 1906-1904, 2019.

BERRINGTON, A. Childlessness in the UK. In: KREYENFELD, M.; KONIETZKA, D. (ed.). Childlessness in Europe: contexts, causes, and consequences. Cham, Switzerland: Springer International Publishing, 2017. p. 57-76. (Demographic Research Monographs). 
BERRINGTON, A.; PATTARO, S. Educational differences in fertility desires, intentions and behaviour: a life course perspective. Advances in Life Course Research, v. 21, p. 10-27, Sep. 2014.

BINSTOCK, G.; CABELLA, W. Las mujeres que terminan su vida reproductiva sin hijos: evolución reciente en América Latina (1980-2010). In: XII CONGRESO DE LA ASOCIACIÓN DE DEMOGRAFÍA HISTÓRICA. Anais [...]. Porto: ADEH, 2019

BUHR, P.; HUININK, J. Why childless men and women give up on having children. European Journal of Population, v. 33, n. 4, p. 585-606, 2017.

BUSETTA, A.; MENDOLA, D.; VIGNOLI, D. Persistent joblessness and fertility intentions. Demographic Research, v. 40, p. 185-218, Jan. 2019.

CABELLA, W.; NATHAN, M.; PARDO, I. La caída de la fecundidad en Uruguay entre 2015 y 2018. In: FONDO DE POBLACIÓN DE LAS NACIONES UNIDAS; MINISTERIO DE SALUD PÚBLICA; UNIVERSIDAD DE LA REPÚBLICA. Descenso acelerado de la fecundidad en Uruguay entre 2015 y 2018: tres estudios para su análisis. Montevideo: UNFPA, MSPy Universidad de la República, 2019. p. 33-70.

CABELLA, W. Dissoluções e formação de novas uniões: uma análise demográfica das tendências recentes no Uruguai. Tese (Doutorado em Demografia) - Instituto de Filosofia e Ciências Humanas, Universidade Estadual de Campinas (Unicamp), Campinas, 2006.

CASTERLINE, J. B.; EL-ZEINI, L. O. The estimation of unwanted fertility. Demography, v. 44, n. 4, p. 729-745, 2007.

CAVENAGHI, S.; DINIZ ALVES, J. Childlessness in Brazil: socioeconomic and regional diversity. In: XXVII IUSSP INTERNATIONAL POPULATION CONFERENCE. Proceedings [...]. Busan, Republic of Korea: IUSSP, 2013. Disponível em: http://iussp.org/sites/default/files/event_call_for_papers/ ChildlessNessBrazil_Final.pdf.

CRAIG, B. M. et al. A generation of childless women: lessons from the United States. Women's Health Issues, v. 24, n. 1, p. e21-e27, 2014.

ESPING-ANDERSEN, G.; BILLARI, F. C. Re-theorizing family demographics. Population and Development Review, v. 41, n. 1, p. 1-31, 2015.

ESTEVE, A.; TREVIÑO, R. Los grandes porqués de la (in)fecundidad en España. Perspectives Demogràfiques, v. 13, p. 1-4, 2019.

FANTA GARRIDO, J.; SACCO, N. Tendencias de nuliparidad definitiva en países de América Latina y el Caribe: ¿hacia la desuniversalización de la maternidad? Coyuntura Demográfica, v. 13, p. 25-31, 2018.

FERNÁNDEZ SOTO, M. Estudio sobre las trayectorias conyugales de las mujeres del Gran Montevideo. Revista Latinoamericana de Población, Ano 4, n. 7, p. 79-104, enero/diciembre 2010.

FERNÁNDEZ SOTO, M. El efecto de las disoluciones conyugales en la fecundidad de las mujeres de Montevideo. Tesis (Doctorado en Ciencias Sociales con Especialización en Estudios de Población) - Facultad de Ciencias Sociales, Universidad de la República, Montevideo, 2019.

GEMMILL, A. From some to none? Fertility expectation dynamics of permanently childless women. Demography, v. 56, n. 1, p. 129-149, 2019.

GILLESPIE, R. Childfree and feminine: understanding the gender identity of voluntarily childless women. Gender and Society, v. 17, n. 1, p. 122-136, 2003.

GRAHAM, M. Is being childless detrimental to a woman's health and well-being across her life course? Women's Health Issues, v. 25, n. 2, p. 176-184, 2015.

GUZZO, K. B. New partners, more kids: multiple-partner fertility in the United States. The Annals of the American Academy of Political and Social Science, v. 654, n. 1, p. 66-86, jul. 2014. 
HAKIM, C. A new approach to explaining fertility patterns: preference theory. Population and Development Review, v. 29, n. 3, p. 349-374, 2003.

HAYFORD, S. R. The evolution of fertility expectations over the life course. Demography, v. 46, n. 4, p. 765-783, 2009.

HAYFORD, S. R. Marriage (still) matters: the contribution of demographic change to trends in childlessness in the United States. Demography, v. 50, n. 5, p. 1641-1661, 18 out. 2013.

HUIJTS, T.; KRAAYKAMP, G.; SUBRAMANIAN, S. V. Childlessness and psychological well-being in context: a multilevel study on 24 European countries. European Sociological Review, v. 29, n. 1, p. 32-47, 2013.

IACOVOU, M.; TAVARES, L. P. Yearning, learning and conceding: (some of) the reasons people change their childbearing intentions. Population and Development Review, v. 37, n. 1, p. 89123, 2011.

JALOVAARA, M. et al. Education, Gender, and cohort fertility in the Nordic countries. European Journal of Population, 2018.

JALOVAARA, M.; FASANG, A. E. From never partnered to serial cohabitors: union trajectories to childlessness. Demographic Research, v. 36, p. 1703-1720, 25 maio 2017.

KELLY, M. Women's voluntary childlessness: a radical rejection of motherhood? Women's Studies Quarterly, v. 37, p. 157-172, 2009.

KREYENFELD, M.; KONIETZKA, D. (ed.). Childlessness in Europe: contexts, causes, and consequences. Cham, Switzerland: Springer International Publishing, 2017.

KUHNT, A. K.; TRAPPE, H. Channels of social influence on the realization of short-term fertility intentions in Germany. Advances in Life Course Research, v. 27, p. 16-29, 2016.

LIMA, E.; ZEMAN, K.; SOBOTKA, T. Twentieth century changes in family size in Latin America Analyses through cohort fertility and parity progression keywords. In: ANNUAL MEETING OF THE POPULATION ASSOCIATION OF AMERICA. Proceedings [...]. Austin, Texas: Population Association of America, 2018.

MERZ, E. M.; LIEFBROER, A. C. The attitude toward voluntary childlessness in Europe: cultural and institutional explanations. Journal of Marriage and Family, v. 74, n. 3, p. 587-600, 2012.

MIETTINEN, A. et al. Increasing childlessness in Europe: time trends and country differences. [S.I.]: European Union's Seventh Framework Programme, 2015. (Families And Societies Working Paper Series, 33).

MILLS, M. et al. Gender equity and fertility intentions in Italy and the Netherlands. Demographic Research, v. 18, n. 29, p. 1-26, 2008.

MILLS, M. et al. Why do people postpone parenthood? Reasons and social policy incentives. Human Reproduction Update, v. 17, n. 6, p. 848-860, 2011.

MYNARSKA, M. et al. Diverse paths into childlessness over the life course. Advances in Life Course Research, v. 25, p. 35-48, 2015.

NATHAN, M. La creciente heterogeneidad en la edad al primer hijo en el Uruguay: un análisis de las cohortes de 1951 a 1990. Notas de Población, v. 100, p. 35-59, 2015.

NATHAN, M.; PARDO, I.; CABELLA, W. Diverging patterns of fertility decline in Uruguay. Demographic Research, v. 34, n. 20, p. 563-586, 2016. 
NEYER, G.; HOEM, J. M.; ANDERSSON, G. Education and childlessness: the influence of educational field and educational level on childlessness among Swedish and Austrian women. In: KREYENFELD, M.; KONIETZKA, D. (ed.). Childlessness in Europe: contexts, causes, and consequences. Cham, Switzerland: Springer International Publishing, 2017. p. 183-207.

NÍ BHROLCHÁIN, M.; BEAUJOUAN, E.; BERRINGTON, A. Stability and change in fertility intentions in Britain, 1991-2007. Population Trends, v. 141, n. 1, p. 13-35, 2010.

PELLEGRINO, A.; POLLERO, R. Fecundidad y situación conyugal en el Uruguay. Un análisis retrospectivo. In: CELTO, D.; MIRÓ, C.; SÁNCHEZ ALBORNOZ, N. Cambios demográficos en América Latina: la experiencia de cinco siglos.Córdoba: Universidad Nacional de Córdoba/IUSSP, 1998. p. 229-250.

PELLEGRINO, A. Vida conyugal y fecundidad en la sociedad uruguaya del siglo XX: una visión desde la demografía. In: BARRÁN, J. P.; CAETANO, G. Y.; PORZECANSKI, T. (ed.). Historias de la vida privada en el Uruguay III. Individuo y soledades (1920-1990). Montevideo: Taurus, 1997.

PETERSON, H. Fifty shades of freedom. Voluntary childlessness as women's ultimate liberation. Women's Studies International Forum, v. 53, p. 182-191, 2015.

REHER, D.; REQUENA, M. Was there a mid-20th century fertility boom in Latin America? Revista de Historia Económica, v. 32, n. 3, p. 319-350, 2014.

REHER, D. S. Family ties in western Europe: persistent contrasts. Population and Development Review, v. 24, n. 2, p. 203-234, 1998.

RODMAN, H. The lower-class value stretch. Social Forces, v. 42, n. 2, p. 205, 1963.

ROWLAND, D. T. Historical trends in childlessness. Journal of Family Issues, v. 28, n. 10, p. 13111337, 2007.

SOBOTKA, T. Shifting parenthood to advanced reproductive ages: trends, causes and consequences. In: TREMMEL, J. (ed.). A young generation under pressure? [S.I.]: Springer Berlin Heidelberg, 2010. p. 129-154.

SOBOTKA, T. Childlessness in Europe: reconstructing long-term trends among women born in 1900-1972. In: KREYENFELD, M.; KONIETZKA, D. (eds.). Childlessness in Europe: contexts, causes, and consequences. Cham, Switzerland: Springer International Publishing, 2017. p. 17-53. (Demographic Research Monographs).

SOBOTKA, T.; BEAUJOUAN, É. Two is best? The persistence of a two-child family ideal in Europe. Population and Development Review, v. 40, n. 3, p. 391-419, 2014.

TANTURRI, M. L. et al. State-of-the-art report Childlessness in Europe. [S.l.]: European Union's Seventh Framework Programme, 2015. (Families And Societies Working Paper Series, 32).

TE VELDE, E. et al. The effect of postponement of first motherhood on permanent involuntary childlessness and total fertility rate in six European countries since the 1970s. Human Reproduction, v. 27, n. 4, p. 1179-1183, 2012.

TOCCHIONI, V. Exploring the childless universe: profiles of women and men without children in Italy. Demographic Research, v. 38, n. 19, p. 451-470, 2018.

VAN BAVEL, J.; KOK, J. Pioneers of the modern lifestyle? Childless couples in the early-twentiethcentury Netherland. Social Science History, v. 34, n. 1, p. 47-72, 2010.

WAGNER, M.; HUININK, J.; LIEFBROER, A. C. Running out of time? Understanding the consequences of the biological clock for the dynamics of fertility intentions and union formation. Demographic Research, v. 40, p. 1-26, Jan. 2019. 


\title{
Sobre los autores
}

Ignacio Pardo es doctor en Sociología por la Universidad Complutense de Madrid. Es investigador y docente del Programa de Población de la Facultad de Ciencias Sociales de la Universidad de la República, Uruguay.

Wanda Cabella es doctora en Demografía por la Universidad Estadual de Campinas. Es investigadora y docente del Programa de Población de la Facultad de Ciencias Sociales de la Universidad de la República, Uruguay.

Mathias Nathan es candidato a doctor en Demografía y Estudios de Población, de la Facultad de Ciencias Sociales de la Universidad de la República, Uruguay. Es docente e investigador del Programa de Población de la misma Facultad.

\section{Dirección para correspondencia}

\author{
Ignacio Pardo \\ Programa de Población, Unidad Multidisciplinaria \\ Facultad de Ciencias Sociales, Universidad de la República \\ Constituyente 1502 \\ 11200 - Montevideo, Uruguay \\ Wanda Cabella \\ Programa de Población, Unidad Multidisciplinaria \\ Facultad de Ciencias Sociales, Universidad de la República \\ Constituyente 1502 \\ 11200 - Montevideo, Uruguay \\ Mathias Nathan \\ Programa de Población, Unidad Multidisciplinaria \\ Facultad de Ciencias Sociales, Universidad de la República \\ Constituyente 1502 \\ 11200 - Montevideo, Uruguay
}

\section{Resumo}

\section{As trajetórias das mulheres sem filhos no Uruguai}

O objetivo deste artigo é estudar as mulheres uruguaias que terminam sua vida reprodutiva sem filhos. Além de quantificar o fenômeno, procura-se responder às seguintes questões: quais são seus principais traços socioeconômicos? Quais são as trajetórias típicas que levam essas mulheres a não se tornarem mães? Até que ponto essa situação é o resultado de aspirações reprodutivas frustradas e até que ponto da vontade de não ter filhos? A estratégia metodológica é baseada na combinação de informações quantitativas e qualitativas, utilizando métodos mistos. Os resultados evidenciam que a proporção de mulheres nulíparas, ao final do ciclo reprodutivo, diminuiu de aproximadamente $25 \%$ para 10\%, entre as nascidas em 1900-1960, sendo que nos dados mais recentes é detectado um aumento incipiente. Além disso, há uma maior proporção de mulheres sem filhos entre as mulheres de alto nível educacional. Por fim, a tipologização das trajetórias que levam à nuliparidade definitiva reafirma a heterogeneidade de caminhos e atributos: embora tenham sido identificadas trajetórias guiadas pela intenção 
de não ter filhos, um importante grupo de mulheres chega ao final da vida reprodutiva como uma consequência não intencional do adiamento da maternidade.

Palavras-chave: Fecundidade. Nuliparidade definitiva. Uruguai.

\begin{abstract}
Life-course trajectories of childless women in Uruguay

This paper aims to analyze Uruguayan women who end their reproductive life span without having children. In addition to quantifying the phenomenon, it seeks to answer the following questions: Which are their main socioeconomic traits? What are the typical trajectories that lead to childlessness? To what extent is it the outcome of unsatisfied reproductive aspirations and to what extent of the will to not have any children? We use a mixed methods approach integrating quantitative and qualitative data. Our results show that the proportion of childless women dropped from approximately $25 \%$ to $10 \%$ among those born in 1900-1960, and that the most recent data show an incipient increase. In addition, there is a higher proportion of childless women among those with a high level of education. Finally, our typology of trajectories towards definitive childlessness confirms the diversity of paths and traits: although trajectories guided by the intention of not having children were identified, a significant number of women reach childlessness as an unintended consequence of the postponement of motherhood.
\end{abstract}

Keywords: Fertility. Childlessness. Uruguay.

Recebido para publicação em 25/12/2019 Aceito para publicação em 30/03/2020 